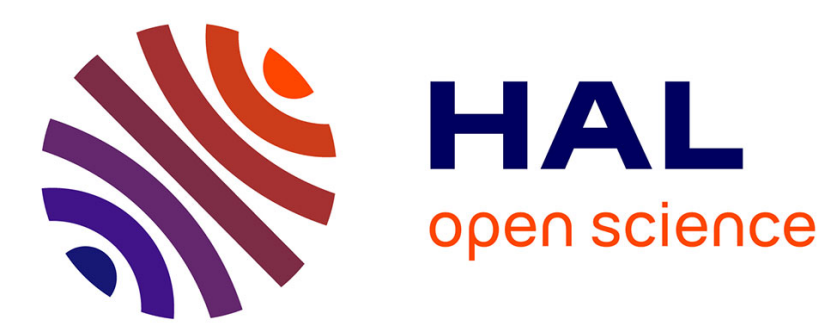

\title{
Nonlinear Robust and Optimal Control via Proper Orthogonal Decomposition
}

\author{
Didier Georges
}

\section{To cite this version:}

Didier Georges. Nonlinear Robust and Optimal Control via Proper Orthogonal Decomposition. NOLCOS 2013 - 9th IFAC Symposium on Nonlinear Control Systems, Sep 2013, Toulouse, France. hal00834339

\section{HAL Id: hal-00834339 \\ https://hal.science/hal-00834339}

Submitted on 14 Jun 2013

HAL is a multi-disciplinary open access archive for the deposit and dissemination of scientific research documents, whether they are published or not. The documents may come from teaching and research institutions in France or abroad, or from public or private research centers.
L'archive ouverte pluridisciplinaire HAL, est destinée au dépôt et à la diffusion de documents scientifiques de niveau recherche, publiés ou non, émanant des établissements d'enseignement et de recherche français ou étrangers, des laboratoires publics ou privés. 


\title{
Nonlinear Robust and Optimal Control via Proper Orthogonal Decomposition
}

\author{
Didier Georges* \\ * Grenoble INP / Univ Grenoble 1 / Univ Grenoble 3 / CNRS, \\ GIPSA-lab, F-38402 Saint Martin d'Hères, \\ (e-mail: didier.georges@gipsa-lab.grenoble-inp.fr).
}

\begin{abstract}
In this paper, a methodology based on proper orthogonal decomposition (POD) for computing approximate closed-loop optimal or robust control laws for finite-dimensional nonlinear systems is proposed. The solution can be obtained on any arbitrary domain of the state space represented by a multidimensional grid. The method only requires the computation of a limited number of well-conditioned two-point boundary value problems and a simple backwards integration of a reduced model of the Hamilton-Jacobi-Bellman or Hamilton-JacobiIsaacs equation associated to the control problem. Two illustrative examples demonstrate the effectiveness of the approach.
\end{abstract}

Keywords: Robust control, optimal control, proper orthogonal decomposition, Hamilton Jacobi - Bellman(Isaacs) equation, Galerkin method.

\section{INTRODUCTION}

Closed-loop computation of nonlinear robust or optimal control problems arising from the Hamilton-Jacobi(Bellmann or Isaacs) equation $(\mathrm{HJ}(\mathrm{B} / \mathrm{I})$ equation) remains a challenging issue, due to the fact that generic solutions are not available for state-space dimensions greater than 1 , and $\mathrm{HJ}(\mathrm{B} / \mathrm{I})$ computation is limited by the curse of dimensionality. In this paper, an approach based on the so-called proper orthogonal decomposition (POD) is proposed. POD seems to have been first proposed by Pearson (1901) and is now extensively used for the purpose of finite-dimensional modeling and control of fluids partial differential equations (Ly (2002)), model reduction of nonlinear systems (Lall (2002)), approximate solution of infinite-dimensional optimal control problems (Kunisch (2008)). POD provides a nice way to get orthonormal basis functions from a finite number of experiments (called "snapshots"), i.e. system solutions obtained by simulations or observations, which optimally span a subspace of the system space. A way to compute approximate solutions to $\mathrm{HJ}(\mathrm{B} / \mathrm{I})$ equations by using functional basis has been proposed in Georges (1996) and Beard (1998). The goal of this paper is to come back to this idea using POD rather than a standard polynomial basis which appears to be illconditioned. In this paper, some preliminary results will be provided: first the robust and optimal control approximate solutions of a very simple one-dimensional example, which gives a nice insight to the proposed approach, are studied. Secondly, an example of practical interest is studied: it consists in the computation of the POD approximate solution of the nonlinear optimal regulator of a boost converter.

The paper is organized as follows. In section 2 , some background is provided on proper orthogonal decomposition. Section 3 describes the overall methodology based on POD for solving $\mathrm{HJ}(\mathrm{B} / \mathrm{I})$ equations. In section 4, two illustrative examples demonstrate the effectiveness of the approach. Some conclusions and perspectives are given in section 5 .

\section{SOME BACKGROUND ON PROPER ORTHOGONAL DECOMPOSITION}

Let $\left\{E_{i}(x): 1 \leq i \leq N ; x \in \Omega \subset \mathbb{R}^{n}\right\}$ denote the set of $N$ observations (snapshots) of some distributed parameter systems over a domain $\Omega$. These observations can be obtained apart from numerical simulations at $N$ time samples $i=1, \ldots, N$ for instance. The problem is now to determine some basis functions $\left\{\phi_{k}(x)\right\}_{k=1}^{N}$, solutions to the following least-square problem

$$
\min _{\phi_{k}, k=1, \ldots, N} \sum_{i=1}^{N}\left\|E_{i}(x)-\sum_{k=1}^{N}\left\langle E_{i}(x), \phi_{k}(x)\right\rangle \phi_{k}(x)\right\|^{2}
$$

subject to $\left\langle\phi_{i}(x), \phi_{j}(x)\right\rangle=\delta_{i j}$ (orthonormality constraint), where $\langle h, g\rangle=\int_{\Omega} f^{T} g d x$ defines the associated inner product.

It can be easily shown (by simply using Lagrange necessary conditions for optimality) that the solution of this problem is the following eigenvalue problem

$$
\begin{array}{r}
\mathcal{R} \phi_{k}(x):=\sum_{i=1}^{N}\left\langle E_{i}(x), \phi_{k}(x)\right\rangle E_{i}(x)=\lambda_{k} \phi_{k}(x), \\
k=1, \ldots, N \\
\left\langle\phi_{k}(x), \phi_{l}(x)\right\rangle=\delta_{k l},
\end{array}
$$

where $\lambda_{k}$ is nothing but the Lagrange multiplier associated to constraint $\left\langle\phi_{k}(x), \phi_{k}(x)\right\rangle=1$.

$\mathcal{R}$ may be rewritten as a $N \times N$ matrix with the elements

$$
\mathcal{R}_{i j}=\left\langle E_{i}(x), E_{j}(x)\right\rangle,
$$


and solving the symmetric eignevalue problem

$$
\mathcal{R} V_{k}=\lambda_{k} V_{k}, 1 \leq k \leq N, \lambda_{1} \geq \lambda_{2} \geq \ldots \geq \lambda_{N},
$$
with the eigenvectors $V_{k}=\left(a_{1}^{k}, \ldots, a_{N}^{k}\right)^{T}$. Then the basis functions $\phi_{k}(x), k=1, \ldots, N$ may be expressed by

$$
\phi_{k}(x)=\frac{1}{\sqrt{\lambda_{k}}} \sum_{i=1}^{N} a_{i}^{k} E_{i}(x),
$$

Clearly, each basis function is simply a linear combination of the set of experiments. Furthermore, it can be easily shown that the $\phi_{k}(x)$ 's form an orthonormal basis $\left(\left\langle\phi_{k}(x), \phi_{l}(x)\right\rangle=\delta_{k l}\right)$.

By considering only the $K$ most significant eigenvalues of the problem (for instance eigenvalues such that $\left.\sum_{i=1}^{K} \lambda_{i} / \sum_{j=1}^{N} \lambda_{j}>p\right)$, we get a representation of $p \%$ of the energy contained in the experimental set. In practice, we can often observe that $K \ll N$, what allows important basis reduction.

\section{APPROXIMATION OF NONLINEAR OPTIMAL AND ROBUST CONTROL PROBLEM SOLUTIONS VIA POD}

The main goal of this section is to provide a methodology for solving PDEs associated to nonlinear optimal or robust control problems.

\subsection{Some Background on Nonlinear Optimal Control}

Now we consider some nonlinear systems defined by

$$
\dot{x}(t)=F(x(t))+G(x(t)) u(t)
$$

where $x(t) \in \mathbb{R}^{n}$ and $u(t) \in \mathbb{R}^{p}$, with $F(0)=0$. $F$ and $G$ are also assumed to be at least continously differentiable.

We consider the following family of optimal control problems $\mathcal{P}(t, x)$ parametrized by initial $t$ and initial state $x(t)=x$ with control horizon $T$, and whose value functional $V(t, x)$ is given by:

$$
V(t, x)=\min _{u(.)} \int_{t}^{T}\left\{l(x(\tau))+\frac{1}{2} u^{T}(\tau) R(x(\tau)) u(\tau)\right\} d \tau(5)
$$

subject to $\dot{x}(\tau)=F(x(\tau))+G(x(\tau)) u(\tau), \tau \in[t, T]$, with $x(t)=x$, and where

$$
\begin{array}{r}
l(x) \geq 0, \forall x \neq 0, l(0)=0, \\
R(x)=R^{T}(x)>0, \forall x,
\end{array}
$$

where $R(x)$ is a $p \times p$ matrix. By using Bellmann principle of optimality (dynamic programming) and under the assumption that $V(t, x)$ is continuously differentiable, solution to problem $\mathcal{P}(t, x)$ is given by the following HJB equation

$$
\begin{aligned}
\frac{\partial V}{\partial t}(t, x)+\min _{u(.)} H\left(x, \frac{\partial V}{\partial x}\right) & =0, \\
V(T, x) & =0
\end{aligned}
$$

where $H\left(x, \frac{\partial V}{\partial x}\right)=\frac{\partial V}{\partial x}(F(x)+G(x) u)+l(x)+\frac{1}{2} u^{T} R(x) u$ is the so-called Hamiltonian associated to the problem.
By using first order necessary condition for optimality, an optimal solution $u^{*}($.$) is such that$

$$
\begin{array}{r}
u^{*}(\tau)=-R^{-1}(x(\tau)) G^{T}(x(\tau)) \frac{\partial V^{T}}{\partial x} \quad(\tau, x(\tau)), \\
\tau \in[t, T] .
\end{array}
$$

Since $\frac{\partial V}{\partial t}(t, x)+\min _{u(.)} H\left(x, \frac{\partial V}{\partial x}\right)=0$ should hold for all $x \in \mathbb{R}^{n}$, the following necessary condition must be satisfied

$$
\frac{\partial}{\partial x}\left(\frac{\partial V}{\partial t}\right)(t, x)+\min _{u(.)} \frac{\partial}{\partial x}\left(H\left(x, \frac{\partial V}{\partial x}\right)\right)=0
$$

By setting $p(t, x)=\frac{\partial V^{T}}{\partial x}(t, x),(11)$ may be rewritten as the following quasi-linear hyperbolic equation

$$
\begin{array}{r}
\frac{\partial p}{\partial t}+\frac{\partial p}{\partial x}\left(F(x)+G(x) u^{*}\right)+\nabla_{x} l(x) \\
+\frac{\partial}{\partial x}\left[F(x)+G(x) u^{*}\right]^{T} p=0 \\
p(T, x)=0 .
\end{array}
$$

$p(t, x)$ is nothing but the adjoint state associated to the problem. Solution to this hyperbolic PDE may be obtained by the method of characteristics. Not surprisingly, the characteristic lines are solutions of the canonical equations associated to $\mathcal{P}(t, x)$

$$
\begin{array}{r}
\dot{x}=F(x)+G(x) u^{*}, x(t)=x \\
\dot{p}=-\nabla_{x} l(x)-\frac{\partial}{\partial x}\left[F(x)+G(x) u^{*}\right]^{T} p, p(T)=0
\end{array}
$$

where $u(.)^{*}$ is given by $(10)$. The characteristic equations are the canonical equations associated to problem $\mathcal{P}(t, x)$ which constitues a two-point boundary value problem (denoted $T P B V P(t, x)$ in what follows).

When $T \rightarrow+\infty$, we get the so-called optimal regulator problem (Moylan (1973)), whose solution $V(x)$ is given by the following algebraic HJB equation

$$
\min _{u(.)} H\left(x, \frac{\partial V}{\partial x}\right)=0, V(0)=0,
$$

with

$$
V(x)=\min _{u(.)} \int_{0}^{\infty}\left\{l(x(\tau))+\frac{1}{2} u^{T}(\tau) R(x(\tau)) u(\tau)\right\} d \tau(17)
$$
subject to $\dot{x}(\tau)=F(x(\tau))+G(x(\tau)) u(\tau)$, with $x(0)=x$. Similarly (12) becomes

$$
\begin{array}{r}
\frac{\partial p}{\partial x}\left(F(x)+G(x) u^{*}\right)+\nabla_{x} l(x) \\
+\frac{\partial}{\partial x}\left[F(x)+G(x) u^{*}\right]^{T} p=0, \\
p(0)=0,
\end{array}
$$

where $u^{*}=-R^{-1}(x) G^{T}(x) \frac{\partial V}{\partial x}(x)$.

Under system controllability and zero-state observablity of $(l(x, 0), F(x, 0))$ assumptions, solution $V(x)>0$ is a closed-loop Lyapunov functional since $\dot{V}=-l\left(x, u^{*}(x)\right)$ and $u^{*}$ given by $(10)$ is a nonlinear state feedback, thanks to Lasalle's invariance principle. 


\subsection{Some Background on Nonlinear Robust Control}

Extension to robust control with disturbance attenuation (see Didinsky (1993) for instance) (here denoted $\mathcal{P}_{r}(t, x)$ ) is a direct generalization of the previous approach to a two-player zero-sum differential game defined as follows

$$
\begin{array}{r}
V(t, x)=\min _{u(.)} \max _{w(.)} \int_{t}^{T}\left\{l(x(\tau))+\frac{1}{2} u^{T}(\tau) R(x(\tau)) u(\tau)\right. \\
\left.-\frac{1}{2} \gamma^{2}\|w(\tau)\|^{2}\right\} d \tau
\end{array}
$$

subject to

$$
\dot{x}(\tau)=F(x(\tau))+G_{1}(x(\tau)(\tau)) u(\tau)+G_{2}(x(\tau)) w(\tau),
$$

such that $F(0)=0$, with $x(t)=x$, and where $w$ is the disturbance input.

Under regularity assumptions, the solution is obtained from the so-called HJI equation

$$
\begin{array}{r}
\frac{\partial V}{\partial t}(t, x)+\min _{u(.)} \max _{w(.)} H\left(x, \frac{\partial V}{\partial x}\right)=0, \\
V(T, x)=0
\end{array}
$$

where $H\left(x, \frac{\partial V}{\partial x}\right)=\frac{\partial V}{\partial x}\left(F(x)+G_{1}(x) u+G_{2}(x) w\right)+$ $l(x)+\frac{1}{2} u^{T} R(x) u-\frac{1}{2} \gamma^{2}\|w\|^{2}$, with $u^{*}$ and $w^{*}$, solutions to necessary conditions

$$
\begin{gathered}
u^{*}=-R^{-1} G_{1}^{T}(x) \frac{\partial V^{T}}{\partial x} \\
w^{*}=\frac{1}{\gamma^{2}} G_{2}^{T}(x) \frac{\partial V^{T}}{\partial x}
\end{gathered}
$$

When $\gamma \rightarrow+\infty$, problem $\mathcal{P}(t, x)$ is easily recovered. In this sense, HJI equations generalize HJB equations. Consequently, the previous derivations apply; in particular some hyperbolic PDEs are obtained, which are very similar to (12)-(18). When $T \rightarrow+\infty, u^{*}$ is also an optimal nonlinear stabilizing feedback law, and $w^{*}$ is the worstcase disturbance acting on the system. The minimum value of $\gamma, \gamma^{*}$, allowing existence of a solution $V(x)$ to the HJI equation defines the so-called robust optimal control problem. $\gamma$ is closely related to the $H_{\infty}$ norm of linear transfert functions (see Isidori (1992)).

\subsection{Computation of the Snapshot Set}

In this section, the way to get snapshots of $\mathcal{P}(t, x)$ for computing a POD basis is discussed. A similar approach is possible for robust problems $\mathcal{P}_{r}(t, x)$, which will not be detailed in this paper.

Since we are interested in finding a solution in a domain $\Omega \subset \mathbb{R}^{n}$ including the origin, we define a multi-dimensional regular grid on $\prod_{i=1}^{n}\left[a_{i}, b_{i}\right]$. We also define an approximate inner product

$$
\begin{aligned}
\langle f, g\rangle= & \int_{\Omega} f g d x \\
\approx & \sum_{i_{1}=1}^{M_{1}} \sum_{i_{2}=1}^{M_{2}} \ldots \sum_{i_{n}=1}^{M_{n}} f\left(x_{i_{1}}, x_{i_{2}}, \ldots, x_{i_{n}}\right) \times \\
& g\left(x_{i_{1}}, x_{i_{2}}, \ldots, x_{i_{n}}\right) d x_{1} d x_{2} \ldots d x_{n} \\
= & \langle f, g\rangle_{a}
\end{aligned}
$$

where the $M_{i}$ 's and the $d x_{i}$ 's are the number of space samples and the space discretization increment in dimension $i$, respectively. For simplification purpose, we denote $x_{g}$, the set of all the space samples $\left(x_{i_{1}}, x_{i_{2}}, \ldots, x_{i_{n}}\right)$ on the spatial grid.

We also define a grid $\left[t_{1}, t_{2}, . ., t_{N}\right]$ of the time interval $[0, T]$, where the way to choose horizon $T$ will be discussed later.

The snapshot set $\left\{E_{i}\right\}_{i=1, \ldots, N}$ will be defined as the collection of the numerical optimal cost functionals

$$
E_{i}\left(x_{g}\right)=V\left(t_{i}, x_{g}\right)
$$

solutions to $\operatorname{TPBVP}\left(t_{i}, x_{g}\right), i=1, \ldots, N$.

For each space sample $x_{g}^{j}, j=1, \ldots, M_{1} \times M_{2}, \ldots \times M_{n}$, solution to $T P B V P\left(t_{i}, x_{g}^{j}\right)$ may be obtained by computing the initial adjoint state $p\left(t, x_{g}^{j}\right)$ such that final adjoint state satisfies transversality condition

$$
p\left(T, x\left(T ; t, x_{g}^{j}, u^{*}[t, T]\right)\right)=0,
$$

where $p\left(T, x\left(T ; t, x_{g}^{j}, u^{*}[t, T]\right)\right)$ denotes the adjoint state corresponding to final state $x\left(T ; t, x_{g}^{j}, u^{*}[t, T]\right)$ resulting from initial state $x_{g}^{j}$ and optimal control $u(.)^{*}$ defined on interval $[t, T] . x\left(T ; t, x_{g}^{j}, u^{*}[t, T]\right)$ is obtained by standard numerical integration of caracteristic equations (15) or similar in the robust control case.

In this paper, a Gauss-Newton approach has been used, which appears to be very effective to solve this problem, by solving the following optimization problem

$$
\min _{p\left(t, x_{g}\right)} \frac{1}{2}\left\|p\left(T, x\left(T ; t, x_{g}^{j}, u^{*}[t, T]\right)\right)\right\|^{2} .
$$

Finally $E_{i}\left(x_{g}^{j}\right)=V\left(t_{i}, x_{g}^{j}\right)$ will be obtained by integrating the Hamiltonian $H$

$$
V\left(t_{i}, x_{g}^{j}\right)=-\int_{T}^{t_{i}} H\left(x_{g}^{j}, p\left(\tau, x_{g}^{j}\right)\right) d \tau .
$$

It is well known that the computation of optimal two-point boundary value problems is difficult when control horizon $T$ is large due to the fact that the characteristic equations always form an unstable differential system.

In this paper, we consider $T$ sufficiently small to avoid such numerical problems, but large enough, together with a large enough number of snapshots, to provide a sufficient "excitation" of the fundamental modes of the characteristic equations.

Under this fundamental assumption, the computation of any optimal or robust control problem with $T$ large and even $T \rightarrow+\infty$ has been experimentally shown to be possible. 


\subsection{POD Basis Derivation}

Once the previously-described snapshot set is obtained, we are in position to derive a POD basis, by computing the eigenvalues and eignenfunctions of Kernel matrix $\mathcal{R}$, whose coefficients are given by

$$
\begin{aligned}
\mathcal{R}_{i j}= & \left\langle E_{i}\left(x_{g}\right), E_{j}\left(x_{g}\right)\right\rangle_{a}, i, j=1, \ldots, N \\
= & \sum_{i_{1}=1}^{M_{1}} \sum_{i_{2}=1}^{M_{2}} \ldots \sum_{i_{n}=1}^{M_{n}} E_{i}\left(x_{i_{1}}, x_{i_{2}}, \ldots, x_{i_{n}}\right) \times \\
& E_{j}\left(x_{i_{1}}, x_{i_{2}}, \ldots, x_{i_{n}}\right) d x_{1} d x_{2} \ldots d x_{n}
\end{aligned}
$$

The orthonormal basis functions $\phi_{k}(x), k=1, \ldots, N$ may be expressed by

$$
\phi_{k}(x)=\frac{1}{\sqrt{\lambda_{k}}} \sum_{i=1}^{N} a_{i}^{k} E_{i}\left(x_{g}\right),
$$

while the $a_{i}^{k}$ 's are the components of the eigenfunction $V_{k}$ associated to the eigenvalue $\lambda_{k}$.

By considering only the $K$ most significant eigenvalues of the problem (for instance eigenvalues such that $\left.\sum_{i=1}^{K} \lambda_{i} / \sum_{j=1}^{N} \lambda_{j}>p\right)$, we get a representation of $p \%$ of the energy contained in the experimental set.

\subsection{Finite-Dimensional Modeling of $H J(B / I)$ Equations}

The Galerkin method is a powerful tool for finitedimensional modeling and simulation of infinite-dimensional systems (see Fletcher (1984) for instance). In the present case, it consists in seeking an approximate solution $V_{a}\left(t, x_{g}\right)$ to HJB equation (8)-(9) in the POD basis

$$
V_{a}\left(t, x_{g}\right)=\sum_{k=1}^{K} \alpha_{k}(t) \phi_{k}\left(x_{g}\right) .
$$

Introducing this approximation in $\operatorname{PDE}(8)$, and using (10) to compute $u_{a}^{*}$, leads to a residual

$$
\begin{aligned}
R(x, \alpha) & =\frac{\partial V_{a}}{\partial x}\left(F(x)+G(x) u_{a}^{*}\right)+l(x) \\
& +\frac{1}{2} u_{a}^{* T} R(x) u_{a}^{*} .
\end{aligned}
$$

By using the equivalent of a weak formulation, we get a set of $K$ equations

$$
\left\langle R\left(x_{g}, \alpha\right), \phi_{k}\left(x_{g}\right)\right\rangle_{a}=0, k=1, \ldots, K,
$$

where $\alpha$ is the vector of the $\alpha_{k}$ 's, which is equivalent to

$$
\begin{array}{r}
\dot{\alpha}_{k}(t)=-\left\langle\frac { \partial V _ { a } } { \partial x } \left( F\left(x_{g}\right)+\right.\right. \\
\left.+G\left(x_{g}\right) u_{a}^{*}\right)+l\left(x_{g}\right) \\
\left.+\frac{1}{2} u_{a}^{* T} R\left(x_{g}\right) u_{a}^{*}\right\rangle_{a}, \\
k=1, \ldots, K
\end{array}
$$

with $\alpha_{k}(T)=0, k=1, \ldots, K$ (corresponding to terminal condition $V(T, 0)=0)$.
This differential system is integrated backwards for any horizon $T$. The asymptotic solution obviously corresponds to the approximate solution of the optimal regulator $(T \rightarrow$ $+\infty)$.

Some remarks. The storage needed to solve this problem is limited to $N \times \prod_{i=1}^{n} M_{i}$ for the snapshot set, while the one for $\mathcal{R}$ is $N \times N$. Once the computation of the snapshot set is performed, the solution of any problem $\mathcal{P}(t, x)$ is available with any control horizon $T$. The computation may be performed on any arbitrary coarse multidimensional grid. The only additional need will be to carry out some multidimensional interpolation.

\section{SOME ILLUSTRATIVE EXAMPLES}

\subsection{A scalar example}

We consider the optimal and robust control of the following scalar unstable nonlinear system (see Georges (1996))

$$
\dot{x}=x^{3}+u
$$

The optimal infinite-horizon control problem is

$$
\min _{u(.)} \int_{0}^{\infty}\left(x^{2}+u^{2}\right) d t
$$

subject to $(39)$.

The optimal control $u^{*}$ is explicitly given by

$$
u^{*}=-x^{3}-x \sqrt{x^{4}+1} .
$$

leading to the closed-loop globally stable system $\dot{x}=$ $-x \sqrt{x^{4}+1}$

We consider the computation of an approximate solution on domain $\Omega=[-2,2]$. The number of time samples and the number of spatial samples are provided in Table 1 , together with horizon $T$ used for generating the snapshot set. Fig 1 provides a comparison between the true optimal control and the approximate one. As shown by the figure, the fit is excellent (relative error $\left\|u^{*}-u_{a}\right\| /\left\|u^{*}\right\|=2.8 e^{-3}$ ). Fig. 2 presents the snapshot set. Fig 3 shows the five most significant POD basis functions obtained in this case (over 30 possible basis functions), which have been used to generate the approximate $V_{a}\left(t, x_{g}\right)$. Fig. 4 demonstrates the effectiveness of the approach when a coarser grid in both time and space is used $(N=5$ and $M=11$, relative error $\left.\left\|u^{*}-u_{a}\right\| /\left\|u^{*}\right\|=1.5 e^{-3}\right)$.

The optimal robust control problem with disturbance attenuation is defined by

$$
\min _{u(.)} \int_{0}^{\infty}\left(x^{2}+u^{2}-\gamma^{2} w^{2}\right) d t
$$

subject to $\dot{x}=x^{3}+u+w$, with $\gamma=1^{+}$corresponding to the optimal attenuation factor. The explicit solution $u^{*}$ is given by

$$
u^{*}=-\left(x^{3}+x \sqrt{x^{4}+1-\frac{1}{\gamma^{2}}}\right) /\left(1-\frac{1}{\gamma^{2}}\right) .
$$

Notice that when $\gamma \rightarrow+\infty$, optimal control solution (41) is recovered. 
The same approach has been used to compute an approximate solution $u_{a}^{*}$ with the parameters given by Table 2 . Fig. 5 shows a comparison between the true and approximate controls demonstrating a good performance (relative error $\left\|u^{*}-u_{a}\right\| /\left\|u^{*}\right\|=2.7 e^{-3}$ ) for a more difficult problem (see the value of the optimal control for $\gamma^{*}=1.01$, which is closed to the problem singularity).

Table 1. Computation parameters - Optimal control

\begin{tabular}{|c||c|c|}
\hline$N$ & $M$ & $T$ \\
\hline 30 & 31 & 0.1 \\
\hline
\end{tabular}

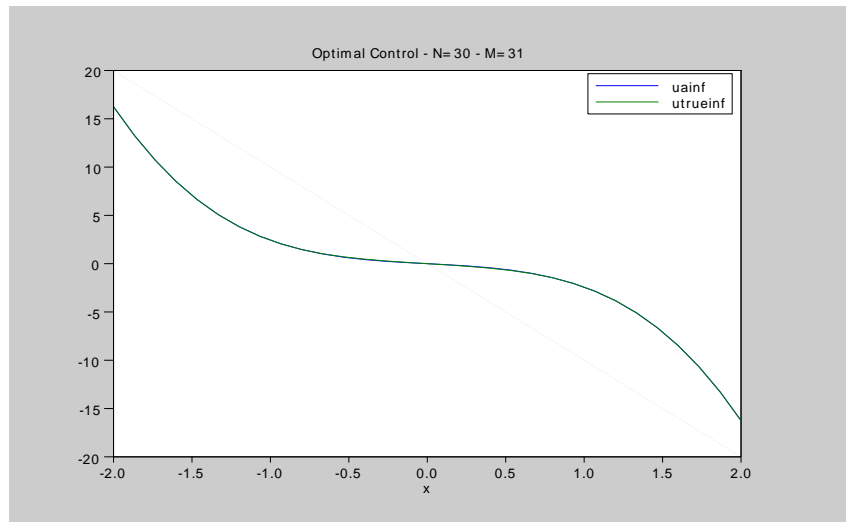

Fig. 1. Comparison between the exact and approximate infinite-horizon optimal controls.

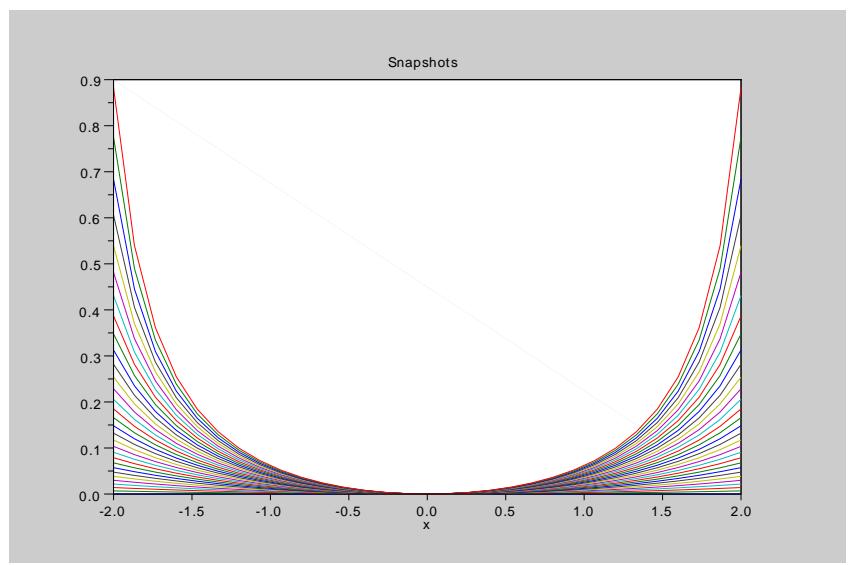

Fig. 2. Snapshot set.

Table 2. Computation parameters - Optimal robust control

\begin{tabular}{|c||c|c|c|}
\hline$N$ & $M$ & $T$ & $\gamma$ \\
\hline 30 & 31 & 0.1 & 1.01 \\
\hline
\end{tabular}

\subsection{Nonlinear optimal regulator for a boost converter}

DC-to-DC boost converters are well-known power electronics devices used to elevate input voltage in many embedded applications (Erickson (2000)). This system exhibits non minimum phase behavior, which renders it difficult to control.

The average model of the boost converter is given by

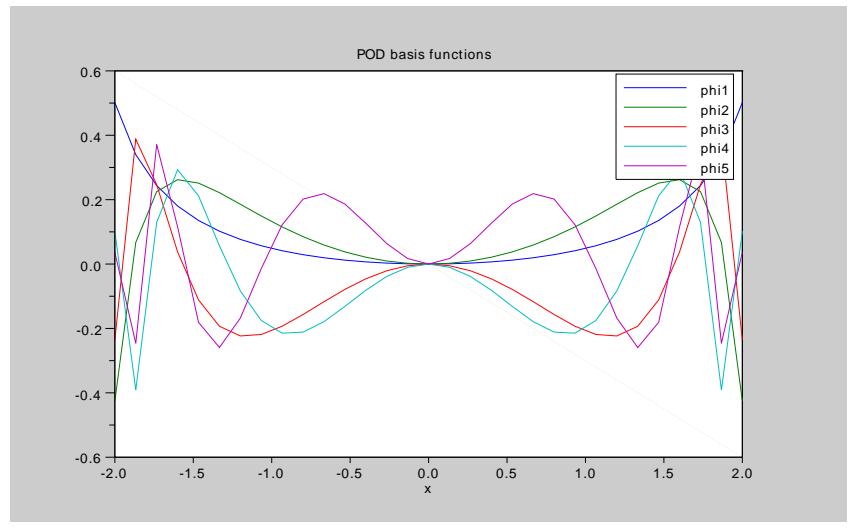

Fig. 3. The five most significant POD basis functions obtained with $p=0.999$.

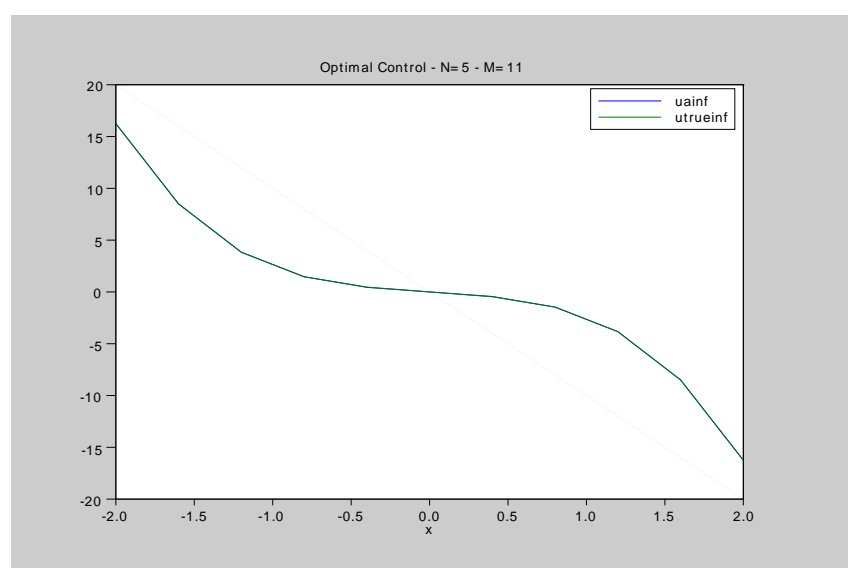

Fig. 4. Comparison between the exact and approximate infinite-horizon optimal controls on a coarse grid.

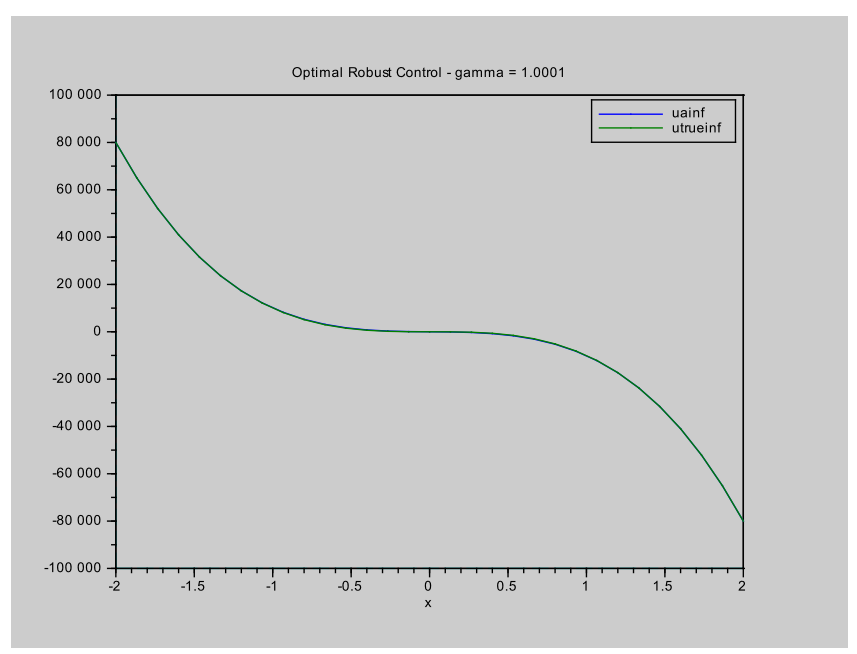

Fig. 5. Comparison between the exact and approximate infinite-horizon optimal robust controls.

$$
\begin{array}{r}
\dot{I}=-u V / L+E / L \\
\dot{V}=u I / C-V /(R C)
\end{array}
$$

where $E$ is the input voltage, $I$ is the inductance current, $V$ is the output voltage, and $u$ the inverse of duty cycle of the converter acting as control input. 
Here we consider the optimal regulation around the equilibrium defined by $\left(I_{e}, V_{e}, u_{e}\right)$ of the boost converter described by Table 3 .

The optimal control problem is defined by

$$
\min _{u} \int_{0}^{\infty}\left(\tilde{V}^{2}+\tilde{u}^{2}\right) d t
$$

subject to the average model error dynamics

$$
\begin{array}{r}
\dot{\tilde{I}}=-u_{e} \tilde{V} / L-V \tilde{u} / L \\
\dot{\tilde{V}}=u_{e} \tilde{I} / C-\tilde{V} /(R C)+I \tilde{u} / C
\end{array}
$$

where $\tilde{I}=I-I_{e}, \tilde{V}=V-V_{e}$, and $\tilde{u}=u-u_{e}$.

Table 3. Boost converter parameters

\begin{tabular}{|c||c||c||c||c||c||c|}
\hline$L$ & $C$ & $R$ & $E$ & $I_{e}$ & $V_{e}$ & $u_{e}$ \\
\hline $2 m H$ & $50 \mu F$ & $R=100 \Omega$ & $1 V$ & $10 V$ & $1 A$ & 0.1 \\
\hline
\end{tabular}

Fig. 6 gives the approximate infinite-horizon optimal control law obtained on domain $D=[-0.5,+0.5] \times$ $[-0.5,+0.5]$. Only 4 POD basis functions have been used with 10 time samples $(N=10)$ and 15 spatial samples in each dimension $\left(M_{1}=M_{2}=15\right)$. A change of time scale $t^{\prime}=1000 t$ has been performed to avoid bad conditioning. Fig. 7 shows the snapshot corresponding to $t^{\prime}=T=5$ and the approximate infinite-horizon Bellman function.

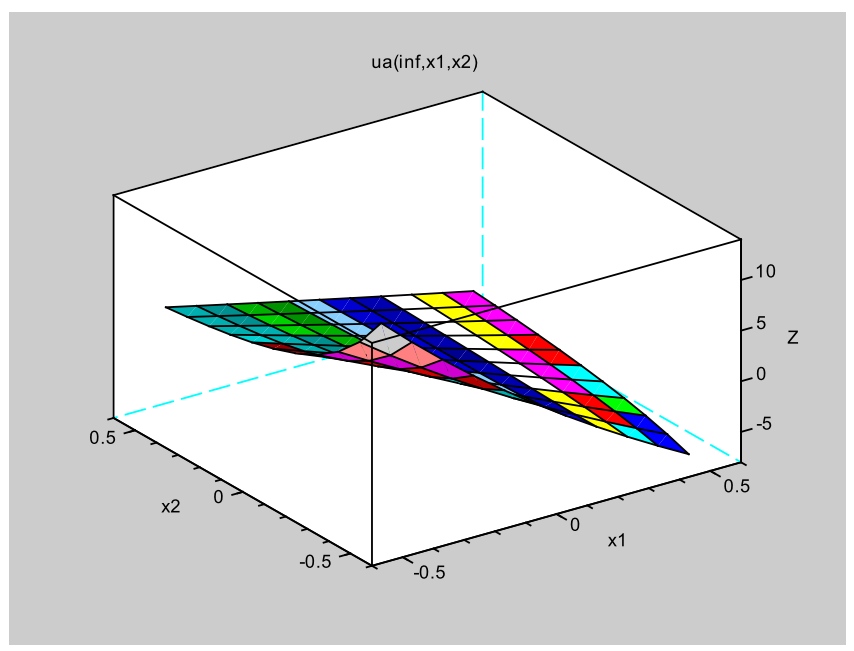

Fig. 6. Approximate infinite-horizon optimal control law of the boost converter.

\section{CONCLUSIONS AND PERSPECTIVES}

Some preliminary results on the computation of optimal or robust nonlinear control problems via POD have been provided in this paper by using a Galerkin method based on a POD basis. The method only requires the computation of a limited number of well-conditioned twopoint boundary value problems and a simple backward integration of a reduced model of the Hamilton-JacobiBellman or Hamilton-Jacobi-Isaacs equation associated to the control problem. Another advantage of the method is its ability to handle coarse grids. A similar approach can be derived for discrete-time nonlinear optimal or robust control problems. Further works should be undertaken to demonstrate the effectiveness of the here-proposed approach for nonlinear systems with higher dimensions.

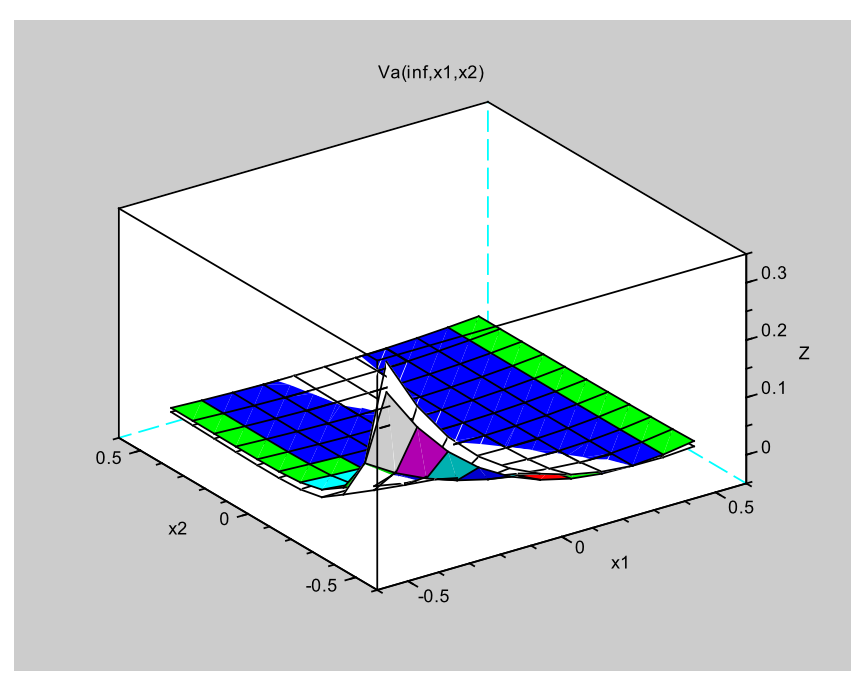

Fig. 7. $V\left(t^{\prime}=2, x\right)$ (uncolored surface) and $V_{a}(\infty, x)$ (colored surface) on $D$.

\section{REFERENCES}

Beard, R.W., Sardis, G.N., and Wen, J.T. (1998). Approximate solutions to the time-invariant HJB equation, J. of Optimal Theory and Applications, 96, 589-626.

Didinsky, G., and Basar, T. (1993). Structural properties of minimax controllers for a class of differential games arising in nonlinear $H_{\infty}$ control, Syst. and Control Letters, 1993, 21, 433-441.

Erickson, R. W., and Maksimovic, D. (2000). Fundamentals of power electronics, Second edition, Kluwer Academic Publishers, ISBN 0-7923-7270-0.

Fletcher C.A.J. (1984). Computational Galerkin methods, Springer Series in Computational Physics. SpringerVerlag, 1984.

Georges, D. (1996). Solutions of nonlinear optimal regulator and $H_{\infty}$ control problems via Galerkin methods, European Journal of Control (1996) 2:211-226.

Isidori, A., and Astolfi, A. (1992). Disturbance attenuation and $H_{\infty}$ control via measurement feedback in nonlinear systems, IEEE Trans. on Autom. Control, AC-37, 12831293.

Kunisch, K., and Volkwien, S. (2008). Proper orthogonal decomposition for optimality systems, ESAIM: M2AN, $42,1,1-23$.

Lall, S., Marsden, J.E., and Glavaski, S. (2002). A subsapce approach to balanced truncation for model reduction of nonlinear control systems, Int. J. Robust Nonlinear Control, 12 (2002) 519-535.

Ly H.V., and Tran H.T. (2002). Proper orthogonal decomposition for flow calculations and optimal control in a horizontal CVD reactor, Quaterly Appl. Math., 60 (2002) 98-110.

Moylan, P.J., and Anderson, B.D.O. (1973). Nonlinear regulator theory and an inverse optimal control problem, IEEE trans. on Autom. Control, AC-18, 460-465.

Pearson, K. (1901). On lines and planes of closest fit to systems of points in space, Phil. Mag., 6th series. 